D) Check for updates

Cite this: Phys. Chem. Chem. Phys., 2017, 19, 14185

DOI: $10.1039 / c 7 c p 90104 f$

\section{Correction: A new potential for methylammonium lead iodide}

\author{
C. M. Handley and C. L. Freeman* \\ Correction for 'A new potential for methylammonium lead iodide' by C. M. Handley et al., Phys. Chem. \\ Chem. Phys., 2017, 19, 2313-2321.
}

rsc.li/pccp

A correction has been made to the table of parameters, Table 1, where unfortunately, a redundant line of parameters, remained within the text. This line "I H2 1142.79 0.025" is not part of this parameter set. The removal of this line of parameters has no impact on the results present within this paper, as this error is only with respect to the parameters as printed within this paper, and not within the input files of the simulations used to generate the results of this paper. We apologise for any confusion this may have incurred.

The Royal Society of Chemistry apologises for these errors and any consequent inconvenience to authors and readers. 
Table 1 Parameterisation

$\underline{\text { Buckingham potentials }\left(A \exp (-\rho / r)-C / r^{6}\right)}$

\begin{tabular}{llllc}
\hline & & $A(\mathrm{eV})$ & $\rho(\AA)$ & $C\left(\mathrm{eV} \AA^{-6}\right)$ \\
\hline $\mathrm{Pb}$ & $\mathrm{I}$ & 6876.2 & 0.32367 & 510.0 \\
$\mathrm{~Pb}$ & $\mathrm{~Pb}$ & 17032.7 & 0.285 & 1100.19 \\
$\mathrm{I}$ & $\mathrm{I}$ & 1791.81 & 0.46529 & 470 \\
\hline
\end{tabular}

Lennard-Jones $12-6$ potential $\left(X / r^{12}-Y / r^{6}\right)$

\begin{tabular}{|c|c|c|c|c|}
\hline & & $X\left(\mathrm{eV} \AA^{12}\right)$ & & $Y\left(\mathrm{eV}^{6}\right)$ \\
\hline $\mathrm{Pb}$ & C & 100000.89 & & 121.072 \\
\hline $\mathrm{Pb}$ & $\mathrm{N}$ & 90000.60 & & 131.856 \\
\hline $\mathrm{Pb}$ & H1 & 1435.31 & & 8.789 \\
\hline $\mathrm{Pb}$ & $\mathrm{H} 2$ & 3590.72 & & 16.445 \\
\hline I & $\mathrm{C}$ & 15180.13 & & 0.743 \\
\hline I & $\mathrm{N}$ & 40465.16 & & 0.809 \\
\hline I & H1 & 1134.51 & & 0.054 \\
\hline I & $\mathrm{H} 2$ & 942.79 & & 0.025 \\
\hline $\mathrm{C}$ & $\mathrm{C}$ & 45262.52 & & 29.32 \\
\hline $\mathrm{C}$ & $\mathrm{N}$ & 43197.02 & & 31.98 \\
\hline $\mathrm{N}$ & $\mathrm{N}$ & 40975.84 & & 34.77 \\
\hline C & H1 & 986.76 & & 2.66 \\
\hline $\mathrm{C}$ & $\mathrm{H} 2$ & 111.38 & & 0.90 \\
\hline $\mathrm{N}$ & H1 & 875.63 & & 2.80 \\
\hline $\mathrm{N}$ & $\mathrm{H} 2$ & 92.25 & & 0.91 \\
\hline H1 & H1 & 8.76 & & 0.15 \\
\hline $\mathrm{H} 1$ & $\mathrm{H} 2$ & 0.40 & & 0.033 \\
\hline $\mathrm{H} 2$ & $\mathrm{H} 2$ & 0.006 & & 0.004 \\
\hline \multicolumn{5}{|c|}{ Two body potentials $\left(\frac{1}{2} k\left(r-r_{0}\right)^{2}\right)$} \\
\hline & & $k\left(\mathrm{eV} \AA^{-2}\right)$ & & $r_{0}(\AA)$ \\
\hline $\mathrm{C}$ & $\mathrm{N}$ & 25.480 & & 1.499 \\
\hline $\mathrm{C}$ & H1 & 29.395 & & 1.091 \\
\hline $\mathrm{N}$ & $\mathrm{H} 2$ & 32.024 & & 1.033 \\
\hline \multicolumn{5}{|c|}{ Three body potentials $\left((k / 2)\left(\theta-\theta_{0}\right)^{2}\right)$} \\
\hline & & & $k(\mathrm{eV})$ & $\theta_{0}\left({ }^{\circ}\right)$ \\
\hline $\mathrm{N}$ & C & $\mathrm{H} 1$ & 4.254 & 108.0 \\
\hline $\mathrm{C}$ & $\mathrm{N}$ & $\mathrm{H} 2$ & 4.009 & 110.11 \\
\hline H1 & $\mathrm{C}$ & H1 & 3.388 & 110.74 \\
\hline $\mathrm{H} 2$ & $\mathrm{~N}$ & $\mathrm{H} 2$ & 3.517 & 108.11 \\
\hline \multicolumn{5}{|c|}{4 body potentials $(A[1+\cos (m \phi-\delta)])$} \\
\hline & & $A(\mathrm{eV})$ & $\delta\left({ }^{\circ}\right)$ & $m$ \\
\hline $\mathrm{H} 1$ & $\mathrm{~N}$ & 0.00675 & 0.0 & 3 \\
\hline
\end{tabular}

Charges

\begin{tabular}{lr}
\hline & $q$ \\
\hline $\mathrm{Pb}$ & 1.263 \\
$\mathrm{I}$ & -0.632 \\
$\mathrm{C}$ & 0.072 \\
$\mathrm{~N}$ & -0.832 \\
$\mathrm{H} 1$ & 0.058 \\
$\mathrm{H} 2$ & 0.405 \\
\hline
\end{tabular}

